\title{
POLY-EULER POLYNOMIALS AND ARAKAWA-KANEKO TYPE ZETA FUNCTIONS
}

\author{
YOSHINORI HAMAHATA
}

Abstract: We introduce poly-Euler polynomials, which generalize Euler polynomials. Various results about them are provided. Furthermore, we introduce zeta functions of Arakawa-Kaneko type, and discuss their properties and the relation with poly-Euler polynomials.

Keywords: polylogarithms, Euler numbers and polynomials, Bernoulli numbers and polynomials, zeta function.

\section{Introduction}

Euler polynomials $E_{n}(x)(n=0,1,2, \ldots)$ are defined by the generating function

$$
\frac{2 e^{x t}}{e^{t}+1}=\sum_{n=0}^{\infty} E_{n}(x) \frac{t^{n}}{n !} .
$$

The first few values are $E_{0}(x)=1, E_{1}(x)=x-1 / 2, E_{2}(x)=x^{2}-x, E_{3}(x)=$ $x^{3}-3 x^{2} / 2+1 / 4$. Let $x>0$. We define the Euler zeta function of Hurwitz type by

$$
\zeta_{E}(s, x)=2 \sum_{n=0}^{\infty} \frac{(-1)^{n}}{(n+x)^{s}}
$$

for $\operatorname{Re}(s)>0$. This function is analytically continued to the whole complex $s$ plane as an entire function. In fact, this follows from the fact that for $\lambda \in \mathbb{R} \backslash \mathbb{Z}$, the Lerch zeta function

$$
L(\lambda, x, s)=\sum_{n=0}^{\infty} \frac{e^{2 \pi i \lambda n}}{(n+x)^{s}}, \quad \operatorname{Re}(s)>0
$$

is analytically continued to the whole complex $s$-plane as an entire function (see $[13,(2.2)])$. It is known that for each non-negative integer $n, \zeta_{E}(-n, x)=$ $E_{n}(x)$.

2010 Mathematics Subject Classification: primary: 11B68; secondary: 11M32, 11M35 
In this article, we introduce poly-Euler polynomials, which generalize Euler polynomials. These poly-Euler polynomials are different from those defined in Son-Kim [15]. Various results about them are provided. Furthermore, we introduce zeta functions of Arakawa-Kaneko type, and discuss their properties and the relation with poly-Euler polynomials. We establish some results to lay the foundation of poly-Euler polynomials and their associated zeta functions. The rest of this paper will be organized as follows: we introduce poly-Euler polynomials and numbers in Section 2 and present basic results. In Section 3, we prove some theorems stated in Section 2. In Section 4, we define zeta functions of ArakawaKaneko type, which are associated to poly-Euler polynomials. In Section 5, using Dirichlet characters, we generalize poly-Euler polynomials, Arakawa-Kaneko type zeta functions, and related results. In Section 6, we prove some theorems stated in Section 5. In Section 7, we make a remark.

\section{Poly-Euler polynomials}

\subsection{Polylogarithms}

For an integer $k$, let $\operatorname{Li}_{k}(x)$ be the formal power series given by

$$
\operatorname{Li}_{k}(x)=\sum_{m=1}^{\infty} \frac{x^{m}}{m^{k}}
$$

If $k$ is a negative integer, for instance $k=-r$, then it converges for $|x|<1$ and equals

$$
\operatorname{Li}_{-r}(x)=\frac{\sum_{j=0}^{r}\left\langle\begin{array}{l}
r \\
j
\end{array}\right\rangle x^{r-j}}{(1-x)^{r+1}},
$$

where the $\left\langle\begin{array}{l}r \\ j\end{array}\right\rangle$ are the Eulerian numbers. The Eulerian number $\left\langle\begin{array}{l}r \\ j\end{array}\right\rangle$ is the number of permutations of $\{1, \ldots, r\}$ with $j$ permutation ascents. One has

$$
\left\langle\begin{array}{l}
r \\
j
\end{array}\right\rangle=\sum_{l=0}^{j+1}(-1)^{l}\left(\begin{array}{c}
r+1 \\
l
\end{array}\right)(j-l+1)^{r} .
$$

See [9] for some properties of Eulerian numbers. We give $\mathrm{Li}_{-r}(x)$ for some $r$ :

$$
\begin{aligned}
\operatorname{Li}_{0}(x) & =\frac{x}{1-x}, & \operatorname{Li}_{-1}(x) & =\frac{x}{(1-x)^{2}}, \\
\operatorname{Li}_{-2}(x) & =\frac{x^{2}+x}{(1-x)^{3}}, & \operatorname{Li}_{-3}(x) & =\frac{x^{3}+4 x^{2}+x}{(1-x)^{4}}, \\
\operatorname{Li}_{-4}(x) & =\frac{x^{4}+11 x^{3}+11 x^{2}+x}{(1-x)^{5}}, & \operatorname{Li}_{-5}(x) & =\frac{x^{5}+26 x^{4}+66 x^{3}+26 x^{2}+x}{(1-x)^{6}} .
\end{aligned}
$$

From (2.2) we immediately deduce that, when $k$ is a negative integer, $\operatorname{Li}_{k}(x)$ is a rational function whose denominator is $(1-x)^{|k|+1}$. Summing up, for an 
integer $k \in \mathbb{Z}$, the formal power series $\operatorname{Li}_{k}(x)$ is the $k$-th polylogarithm if $k \geqslant 1$, and a rational function if $k \leqslant 0$. When $k=1$,

$$
\mathrm{Li}_{1}(x)=-\log (1-x)
$$

\subsection{Poly-Euler polynomials and numbers}

Using $\operatorname{Li}_{k}(x)$, let us introduce poly-Euler polynomials and numbers.

Definition 2.1. For every integer $k$, we define a sequence of polynomials $\left\{E_{n}^{(k)}(x)\right\}$, which we call poly-Euler polynomials, by

$$
\frac{2 \operatorname{Li}_{k}\left(1-e^{-t}\right)}{t\left(e^{t}+1\right)} e^{x t}=\sum_{n=0}^{\infty} E_{n}^{(k)}(x) \frac{t^{n}}{n !} .
$$

The numbers $E_{n}^{(k)}:=E_{n}^{(k)}(0)$ are called poly-Euler numbers.

It is easy to see that for any $n \geqslant 0$,

$$
E_{n}^{(1)}(x)=E_{n}(x)
$$

\section{Remark 2.2.}

(1) We can extend poly-Euler polynomials by

$$
\frac{(1-u) \operatorname{Li}_{k}\left(1-e^{-t}\right)}{t\left(e^{t}-u\right)} e^{x t}=\sum_{n=0}^{\infty} E_{n}^{(k)}(u ; x) \frac{t^{n}}{n !}
$$

for a given real algebraic number $u$.

(2) Our poly-Euler polynomials are different from those of Son-Kim [15]. They define poly-Euler polynomials $\mathbb{E}_{n}^{(k)}(x)(n=0,1,2, \ldots)$ by

$$
-\frac{\operatorname{Li}_{k}\left(1-e^{2}\right)}{e^{t}+1} e^{x t}=\sum_{n=0}^{\infty} \mathbb{E}_{n}^{(k)}(x) \frac{t^{n}}{n !} .
$$

Since

$$
\frac{d}{d t} \operatorname{Li}_{k}\left(1-e^{-t}\right)=\frac{1}{e^{t}-1} \operatorname{Li}_{k-1}\left(1-e^{-t}\right)
$$

if $k \geqslant 1$, then the generating function $\sum_{n=0}^{\infty} E_{n}^{(k)}(x) t^{n} / n$ ! can be written as iterated integrals:

$$
\sum_{n=0}^{\infty} E_{n}^{(k)}(x) \frac{t^{n}}{n !}=\frac{2 e^{x t}}{t\left(e^{t}+1\right)} \underbrace{\int_{0}^{t} \frac{1}{e^{t}-1} \int_{0}^{t} \frac{1}{e^{t}-1} \cdots \int_{0}^{t} \frac{1}{e^{t}-1} t d t d t \cdots d t}_{(k-1) \text {-times }}
$$




\subsection{Some identities}

By (2.5),

$$
\left(\sum_{n=0}^{\infty} E_{n}^{(k)} \frac{t^{n}}{n !}\right) e^{x t}=\sum_{n=0}^{\infty} E_{n}^{(k)}(x) \frac{t^{n}}{n !} .
$$

Hence, $E_{n}^{(k)}(x)$ form the Appell sequence (see [14], Chapter 2). We have therefore the following result.

Theorem 2.3. For $k \in \mathbb{Z}, n \geqslant 0$, we have

$$
\begin{aligned}
E_{n}^{(k)}(x) & =\sum_{m=0}^{n}\left(\begin{array}{c}
n \\
m
\end{array}\right) E_{m}^{(k)} x^{n-m}, \\
E_{n}^{(k)}(x+y) & =\sum_{m=0}^{n}\left(\begin{array}{c}
n \\
m
\end{array}\right) E_{m}^{(k)}(x) y^{n-m}, \\
\frac{d}{d x} E_{n+1}^{(k)}(x) & =(n+1) E_{n}^{(k)}(x) .
\end{aligned}
$$

We can express $E_{n}^{(k)}(x)$ in terms of Euler polynomials.

Theorem 2.4. For $k \in \mathbb{Z}, n \geqslant 0$, we have

$$
E_{n}^{(k)}(x)=\frac{1}{n+1} \sum_{m=0}^{\infty} \frac{1}{(m+1)^{k}} \sum_{j=0}^{m+1}(-1)^{j}\left(\begin{array}{c}
m+1 \\
j
\end{array}\right) E_{n+1}(x-j) .
$$

\subsection{Poly-Euler polynomials with non-positive index}

We introduce two-variable polynomials with non-positive index $-k$ to connect $E_{n}^{(-k)}(x)$ and $E_{k}^{(-n)}(x)$.

Definition 2.5. For $m, n \geqslant 0$, define

$$
F_{n}^{(-m)}(x, y)=\sum_{k=0}^{m}\left(\begin{array}{c}
m \\
k
\end{array}\right) E_{n}^{(-k)}(x) \frac{E_{m-k+1}(y)-E_{m-k+1}(y-1)}{2(m-k+1)} .
$$

In addition, put $F_{n}^{(-m)}:=F_{n}^{(-m)}(0,0)$.

Theorem 2.6 (Symmetric formula).

$$
\begin{aligned}
\sum_{n=0}^{\infty} \sum_{m=0}^{\infty} F_{n}^{(-m)}(x, y) \frac{t^{n}}{n !} \frac{u^{m}}{m !} & =\sum_{n=0}^{\infty} \sum_{m=0}^{\infty} F_{m}^{(-n)}(y, x) \frac{t^{n}}{n !} \frac{u^{m}}{m !} \\
& =\frac{2 e^{x t+y u} e^{t+u}\left(1-e^{-t}\right)\left(1-e^{-u}\right)}{t u\left(e^{t}+1\right)\left(e^{u}+1\right)\left(e^{t}+e^{u}-e^{t+u}\right)}
\end{aligned}
$$

This result yields a duality theorem for poly-Euler polynomials. 
Theorem 2.7 (Duality). For $m, n \geqslant 0$, we have

$$
F_{n}^{(-m)}(x, y)=F_{m}^{(-n)}(y, x)
$$

In particular,

$$
\begin{aligned}
F_{n}^{(-m)} & =F_{m}^{(-n)} \\
\sum_{n=0}^{\infty} \sum_{m=0}^{\infty} F_{n}^{(-m)} \frac{t^{n}}{n !} \frac{u^{m}}{m !} & =\frac{e^{t+u}\left(1-e^{-t}\right)\left(1-e^{-u}\right)}{t u\left(e^{t}+1\right)\left(e^{u}+1\right)\left(e^{t}+e^{u}-e^{t+u}\right)}
\end{aligned}
$$

Theorem 2.8 (Inversion formula). For $m, n \geqslant 0$, we have

$$
E_{n}^{(-m)}(x)=\sum_{k=0}^{m}(-1)^{m-k}\left(\begin{array}{c}
m \\
k
\end{array}\right) F_{n}^{(-k)}(x, y)\left(B_{m-k}(y-1)+B_{m-k}(y)\right)
$$

where $B_{n}(x)$ are the classical Bernoulli polynomials given by the generating function

$$
\frac{t e^{x t}}{e^{t}-1}=\sum_{n=0}^{\infty} B_{n}(x) \frac{t^{n}}{n !}
$$

Theorem 2.9. For $m, n \geqslant 0$, we have

$$
\begin{aligned}
F_{n}^{(-m)}(x, y)= & \frac{1}{2(n+1)(m+1)} \\
& \times \sum_{j=1}^{\min (n+1, m+1)}(j !)^{2}\left(\sum_{k=0}^{n+1} E_{n+1-k}(x)\left(\begin{array}{c}
n+1 \\
k
\end{array}\right)\left\{\begin{array}{l}
k \\
j
\end{array}\right\}\right) \\
& \left(\sum_{l=0}^{m+1} E_{m+1-l}(x)\left(\begin{array}{c}
m+1 \\
l
\end{array}\right)\left\{\begin{array}{l}
l \\
j
\end{array}\right\}\right)
\end{aligned}
$$

where

$$
\left\{\begin{array}{c}
n \\
m
\end{array}\right\}=\frac{(-1)^{m}}{m !} \sum_{l=0}^{m}(-1)^{l}\left(\begin{array}{c}
m \\
l
\end{array}\right) l^{n}
$$

are the Stirling numbers of the second kind. 


\subsection{A relation with poly-Bernoulli polynomials}

We briefly review poly-Bernoulli polynomials and numbers $([3])$. Let $k$ denote a fixed integer. Poly-Bernoulli polynomials $B_{n}^{(k)}(x)(n=0,1,2, \ldots)$ are defined by the generating function

$$
\frac{\operatorname{Li}_{k}\left(1-e^{-t}\right)}{1-e^{-t}} e^{x t}=\sum_{n=0}^{\infty} B_{n}^{(k)}(x) \frac{t^{n}}{n !} .
$$

Moreover, we call $B_{n}^{(k)}:=B_{n}^{(k)}(0)(n=0,1,2, \ldots)$ poly-Bernoulli numbers, which were introduced in [12], and then investigated in $[1,2,10,11]$. If $k=1$, then

$$
(-1)^{n} B_{n}^{(1)}(-x)=B_{n}(x) \quad(n \geqslant 0) .
$$

The following result yields a relation among poly-Bernoulli and poly-Euler polynomials.

Theorem 2.10. For $k \in \mathbb{Z}$ and $n \geqslant 1$, we have

$$
n E_{n-1}^{(k)}(x)+n E_{n-1}^{(k)}(x+1)=2 B_{n}^{(k)}(x)-2 B_{n}^{(k)}(x-1) .
$$

\section{Proofs of theorems in Section 2}

Proof of Theorem 2.4. We use the generating function for poly-Euler polynomials.

$$
\begin{aligned}
\frac{2 \operatorname{Li}_{k}\left(1-e^{-t}\right) e^{x t}}{t\left(1+e^{t}\right)} & =\sum_{m=0}^{\infty} \frac{\left(1-e^{-t}\right)^{m+1}}{(m+1)^{k}} \cdot \frac{2 e^{x t}}{t\left(1+e^{t}\right)} \\
& =\sum_{m=0}^{\infty} \frac{1}{(m+1)^{k}} \sum_{j=0}^{m+1}(-1)^{j}\left(\begin{array}{c}
m+1 \\
j
\end{array}\right) \cdot \frac{2 e^{(x-j) t}}{t\left(1+e^{t}\right)} \\
& =\sum_{m=0}^{\infty} \frac{1}{(m+1)^{k}} \sum_{j=0}^{m+1}(-1)^{j}\left(\begin{array}{c}
m+1 \\
j
\end{array}\right) \sum_{n=-1}^{\infty} \frac{E_{n+1}(x-j)}{n+1} \frac{t^{n}}{n !}
\end{aligned}
$$

Proof of Theorem 2.6. We need the following result.

\section{Lemma 3.1.}

$$
\sum_{k=0}^{\infty} \operatorname{Li}_{k}\left(1-e^{-t}\right) \frac{u^{k}}{k !}=\frac{e^{t+u}\left(1-e^{-t}\right)}{e^{t}+e^{u}-e^{t+u}}
$$


Proof. As stated in Kaneko [12], it holds that

$$
\sum_{k=0}^{\infty} \sum_{n=0}^{\infty} B_{n}^{(-k)} \frac{t^{n}}{n !} \frac{u^{k}}{k !}=\frac{e^{t+u}}{e^{t}+e^{u}-e^{t+u}}
$$

To prove Theorem 2.6, we note that the left-hand side becomes

$$
\begin{aligned}
& \frac{1}{2} \sum_{n=0}^{\infty} \sum_{m=0}^{\infty} \sum_{k+l=m}\left(\begin{array}{c}
m \\
k
\end{array}\right) E_{n}^{(-k)}(x) \frac{E_{l+1}(y)-E_{l+1}(y-1)}{l+1} \frac{t^{n}}{n !} \frac{u^{m}}{m !} \\
= & \frac{1}{2 u} \sum_{k=0}^{\infty} \sum_{n=0}^{\infty} E_{n}^{(-k)}(x) \frac{t^{n}}{n !} \frac{u^{k}}{k !} \sum_{l=0}^{\infty}\left(E_{l+1}(y)-E_{l+1}(y-1)\right) \frac{u^{l+1}}{(l+1) !} \\
= & \frac{1}{2 u} \sum_{k=0}^{\infty} \frac{2 \mathrm{Li}_{-k}\left(1-e^{-t}\right) e^{x t}}{t\left(e^{t}+1\right)} \frac{u^{k}}{k !} \sum_{l=0}^{\infty}\left(E_{l+1}(y)-E_{l+1}(y-1)\right) \frac{u^{l+1}}{(l+1) !} \\
= & \frac{e^{x t}}{t u\left(e^{t}+1\right)}\left(\sum_{k=0}^{\infty} \operatorname{Li}_{-k}\left(1-e^{-t}\right) \frac{u^{k}}{k !}\right)\left(\sum_{l=0}^{\infty}\left(E_{l}(y)-E_{l+1}(y-1)\right) \frac{u^{l}}{l !}\right) \\
= & \frac{e^{t+u}\left(1-e^{-t}\right) e^{x t}}{t u\left(e^{t}+1\right)\left(e^{t}+e^{u}-e^{t+u}\right)} \cdot \frac{2\left(e^{y u}-e^{(y-1) u}\right.}{e^{u}+1} \quad \text { (by Lemma 3.1). }
\end{aligned}
$$

This is the right-hand side.

Proof of Theorem 2.8. Starting with the right-hand side, we obtain

$$
\sum_{n=0}^{\infty} \sum_{m=0}^{\infty} \sum_{k=0}^{m}(-1)^{m-k}\left(\begin{array}{c}
m \\
k
\end{array}\right) F_{n}^{(-k)}(x, y)\left(B_{m-k}(y-1)+B_{m-k}(y)\right) \frac{t^{n}}{n !} \frac{u^{m}}{m !}
$$

(put $l=m-k)$

$$
\begin{aligned}
& =\left(\sum_{n=0}^{\infty} \sum_{k=0}^{\infty} F_{n}^{(-k)}(x, y) \frac{t^{n}}{n !} \frac{u^{k}}{k !}\right)\left(\sum_{l=0}^{\infty}\left(B_{l}(y-1)+B_{l}(y)\right) \frac{(-u)^{l}}{l !}\right) \\
& =\frac{2 e^{x t+y u} e^{t+u}\left(1-e^{-t}\right)\left(1-e^{-u}\right)}{t u\left(e^{t}+1\right)\left(e^{u}+1\right)\left(e^{t}+e^{u}-e^{t+u}\right)}\left(\frac{(-u) e^{(y-1)(-u)}}{e^{-u}-1}+\frac{(-u) e^{y(-u)}}{e^{-u}-1}\right)
\end{aligned}
$$

(by Theorem 2.6)

$$
\begin{aligned}
& =\frac{2 e^{x t} e^{t+u}\left(1-e^{-t}\right)}{t\left(e^{t}+1\right)\left(e^{t}+e^{u}-e^{t+u}\right)} \\
& =\sum_{n=0}^{\infty} \sum_{m=0}^{\infty} E_{n}^{(-m)}(x) \frac{t^{n}}{n !} \frac{u^{m}}{m !} \quad \text { (by Lemma 3.1) }
\end{aligned}
$$

which gives the left-hand side. 
Proof of Theorem 2.9. From the left-hand side we get with Theorem 2.6,

$$
\begin{aligned}
\sum_{n=0}^{\infty} \sum_{m=0}^{\infty} F_{n}^{(-m)}(x, y) \frac{t^{n}}{n !} \frac{u^{m}}{m !} & =\frac{2 e^{x t+y u} e^{t+u}\left(1-e^{-t}\right)\left(1-e^{-u}\right)}{t u\left(e^{t}+1\right)\left(e^{u}+1\right)\left(e^{t}+e^{u}-e^{t+u}\right)} \\
& =\frac{2 e^{x t+y u}\left(e^{t}-1\right)\left(e^{u}-1\right)}{t u\left(e^{t}+1\right)\left(e^{u}+1\right)\left\{1-\left(e^{t}-1\right)\left(e^{u}-1\right)\right\}} \\
& =\frac{2 e^{x t+y u}}{t u\left(1+e^{t}\right)\left(1+e^{u}\right)} \sum_{j=1}^{\infty}\left(e^{t}-1\right)^{j}\left(e^{u}-1\right)^{j} \\
& =\frac{1}{t u} \sum_{j=1}^{\infty}\left\{\frac{2 e^{x t}}{1+e^{t}}\left(e^{t}-1\right)^{j}\right\}\left\{\frac{2 e^{y u}}{1+e^{u}}\left(e^{u}-1\right)^{j}\right\}
\end{aligned}
$$

Using the identity

$$
\frac{\left(e^{t}-1\right)^{j}}{j !}=\sum_{n=j}^{\infty}\left\{\begin{array}{l}
n \\
j
\end{array}\right\} \frac{t^{n}}{n !}
$$

we obtain

$$
\begin{aligned}
\frac{1}{2 t u} & \sum_{j=1}^{\infty}\left(\sum_{n=0}^{\infty} E_{n}(x) \frac{t^{n}}{n !} j ! \sum_{k=0}^{\infty}\left\{\begin{array}{l}
k \\
j
\end{array}\right\} \frac{t^{k}}{k !}\right)\left(\sum_{m=0}^{\infty} E_{m}(y) \frac{u^{m}}{m !} j ! \sum_{l=0}^{\infty}\left\{\begin{array}{l}
l \\
j
\end{array}\right\} \frac{u^{l}}{l !}\right) \\
= & \frac{1}{2} \sum_{p=0}^{\infty} \sum_{q=0}^{\infty} \sum_{j=1}^{\infty}(j !)^{2}\left(\sum_{k=0}^{p} E_{p-k}(x)\left(\begin{array}{l}
p \\
k
\end{array}\right)\left\{\begin{array}{l}
k \\
j
\end{array}\right\}\right) \\
& \times\left(\sum_{l=0}^{q} E_{q-l}(y)\left(\begin{array}{l}
q \\
l
\end{array}\right)\left\{\begin{array}{l}
l \\
j
\end{array}\right\}\right) \frac{t^{p-1}}{p !} \frac{u^{q-1}}{q !} \\
= & \sum_{n=0}^{\infty} \sum_{m=0}^{\infty} \frac{1}{2(n+1)(m+1)} \sum_{j=1}^{\infty}(j !)^{2}\left(\sum_{k=0}^{n+1} E_{n+1-k}(x)\left(\begin{array}{c}
n+1 \\
k
\end{array}\right)\left\{\begin{array}{l}
k \\
j
\end{array}\right\}\right) \\
& \times\left(\sum_{l=0}^{m+1} E_{m+1-l}(x)\left(\begin{array}{c}
m+1 \\
l
\end{array}\right)\left\{\begin{array}{l}
l \\
j
\end{array}\right\}\right) \frac{t^{n}}{n !} \frac{u^{m}}{m !} \\
= & \sum_{n=0}^{\infty} \sum_{m=0}^{\infty} \frac{1}{2(n+1)(m+1)} \sum_{j=1}^{\infty}(n+1, m+1) \\
& \times\left(\sum_{l=0}^{m+1} E_{m+1-l}(x)\left(\begin{array}{c}
m+1 \\
l
\end{array}\right)\left\{\begin{array}{l}
l \\
j
\end{array}\right\}\right) \frac{t^{n}}{n !} \frac{u^{m}}{m !}
\end{aligned}
$$

Equating coefficients, we obtain the result.

Proof of Theorem 2.10. We compute both sides of

$$
\frac{2 \operatorname{Li}_{k}\left(1-e^{-t}\right)}{t\left(1+e^{t}\right)}\left(1+e^{t}\right) e^{x t} t=\frac{2 \operatorname{Li}_{k}\left(1-e^{-t}\right)}{1-e^{-t}}\left(1-e^{-t}\right) e^{x t}
$$


While the left-hand side is

$$
\frac{2 \operatorname{Li}_{k}\left(1-e^{-t}\right)}{t\left(1+e^{t}\right)} e^{x t} t+\frac{2 \operatorname{Li}_{k}\left(1-e^{-t}\right)}{t\left(1+e^{t}\right)} e^{(x+1) t} t=\sum_{n=1}^{\infty}\left(n E_{n-1}^{(k)}(x)+n E_{n-1}^{(k)}(x+1)\right) \frac{t^{n}}{n !},
$$

the right-hand side is

$$
\frac{2 \operatorname{Li}_{k}\left(1-e^{-t}\right)}{1-e^{-t}} e^{x t}-\frac{2 \operatorname{Li}_{k}\left(1-e^{-t}\right)}{1-e^{-t}} e^{(x-1) t}=\sum_{n=0}^{\infty}\left(2 B_{n}^{(k)}(x)-2 B_{n}^{(k)}(x-1)\right) \frac{t^{n}}{n !}
$$

from which we deduce the result.

\section{Arakawa-Kaneko type zeta functions}

\subsection{Arakawa-Kaneko type zeta functions - basic properties}

Definition 4.1. For $k \in \mathbb{Z}$, set

$$
Z_{E, k}(s, x)=\frac{2}{\Gamma(s)} \int_{0}^{\infty} \frac{\operatorname{Li}_{k}\left(1-e^{-t}\right)}{1+e^{t}} e^{-x t} t^{s-2} d t
$$

the Laplace-Mellin integral. We call it the Arakawa-Kaneko type zeta function for poly-Euler polynomials.

Proposition 4.2. The zeta function $Z_{E, k}(s, x)$ is defined for $\operatorname{Re}(s)>1$ and $x>0$ if $k \geqslant 1$, and for $\operatorname{Re}(s)>1$ and $x>|k|+1$ if $k \leqslant 0$.

Proof. We prove that $Z_{E, k}(s, x)$ converges for each case.

Case $k \geqslant 1$ : for $t \geqslant 0$, we have

$$
\frac{\operatorname{Li}_{k}\left(1-e^{-t}\right)}{1+e^{t}} e^{-x t} t^{s-2} \leqslant \operatorname{Li}_{k}\left(1-e^{-t}\right) e^{-x t} t^{s-2} \leqslant \frac{t^{s-1}}{e^{x t}} .
$$

Case $k=0$ : for $t \geqslant 0$, we have

$$
\frac{\operatorname{Li}_{k}\left(1-e^{-t}\right)}{1+e^{t}} e^{-x t} t^{s-2}=\frac{e^{t}-1}{1+e^{t}} e^{-x t} t^{s-2} \leqslant \frac{t^{s-1}}{e^{x t}} .
$$

Case $k<0$ : for $t \geqslant 0$, using (2.2), we have

$$
\begin{aligned}
\frac{\operatorname{Li}_{k}\left(1-e^{-t}\right)}{1+e^{t}} e^{-x t} t^{s-2} & =\frac{\sum_{j=0}^{|k|-1}\left\langle\begin{array}{c}
|k| \\
j
\end{array}\right\rangle\left(1-e^{-t}\right)^{|k|-j}}{\left(1+e^{t}\right) e^{(x-|k|-1) t}} t^{s-2} \\
& \leqslant \frac{|k| ! \sum_{j=0}^{|k|-1}\left(1-e^{-t}\right)^{|k|-j}}{\left(1+e^{t}\right) e^{(x-|k|-1) t}} t^{s-2} \\
& =\frac{|k| !\left(e^{t}-1\right)\left\{1-\left(1-e^{-t}\right)^{|k|}\right\}}{\left(1+e^{t}\right) e^{(x-|k|-1) t}} t^{s-2}
\end{aligned}
$$


Since $1-\left(1-e^{-t}\right)^{|k|} \leqslant t$ for all $t \geqslant 0$,

$$
\text { R.H.S. } \leqslant \frac{|k| ! t^{s-1}}{e^{(x-|k|-1) t}}
$$

These ensure the convergence of $Z_{E, k}(s, x)$.

Here is a result about the values at non-positive integers.

Theorem 4.3. We assume that $x>0$ if $k \geqslant 1$, and $x>|k|+1$ if $k \leqslant 0$. Then, the function $s \mapsto Z_{E, k}(s, x)$ has analytic continuation to an entire function on the whole complex s-plane and

$$
Z_{E, k}(-n, x)=(-1)^{n} E_{n}^{(k)}(-x)
$$

holds for $n \geqslant 0$.

Proof. We express $Z_{E, k}(s, x)$ as the sum of two integrals:

$$
\begin{aligned}
Z_{E, k}(s, x)= & \frac{2}{\Gamma(s)} \int_{1}^{\infty} \frac{\operatorname{Li}_{k}\left(1-e^{-t}\right)}{1+e^{t}} e^{-x t} t^{s-2} d t \\
& +\frac{2}{\Gamma(s)} \int_{0}^{1} \frac{\operatorname{Li}_{k}\left(1-e^{-t}\right)}{1+e^{t}} e^{-x t} t^{s-2} d t .
\end{aligned}
$$

The first integral converges absolutely for any $s \in \mathbb{C}$ and $x>0$ and cancels at non-positve integers because $1 / \Gamma(s)$ does so. If $\operatorname{Re}(s)>1$, then the second integral can be written as

$$
\frac{1}{\Gamma(s)} \sum_{i=0}^{\infty} \frac{E_{i}^{(k)}(-x)}{i !} \cdot \frac{1}{i+s}
$$

from which we have for a non-negative integer $n$

$$
Z_{E, k}(-n, x)=\left(\lim _{s \rightarrow-n} \frac{1}{\Gamma(s)(n+s)}\right) \frac{E_{n}^{(k)}(-x)}{n !}=(-1)^{n} E_{n}^{(k)}(-x) .
$$

When $k=1$, one obtains the zeta function in (1.1):

$$
\begin{aligned}
Z_{E, 1}(s, x) & =\frac{2}{\Gamma(s)} \int_{0}^{\infty} \frac{e^{-x t}}{e^{t}+1} t^{s-1} d t=2 \sum_{n=1}^{\infty} \frac{(-1)^{n-1}}{(n+x)^{s}} \\
& =\zeta_{E}(s, x+1) .
\end{aligned}
$$

We give two kinds of expressions for $Z_{E, k}(s, x)$.

Theorem 4.4. The zeta function $Z_{E, k}(s, x)$ can be expressed as follows.

(i) If $s \neq 1$, then

$$
Z_{E, k}(s, x)=\frac{1}{s-1} \sum_{m=0}^{\infty} \frac{1}{(m+1)^{k}} \sum_{j=0}^{m+1}(-1)^{j}\left(\begin{array}{c}
m+1 \\
j
\end{array}\right) \zeta_{E}(s-1, x+j+1) .
$$


(ii) If $k \leqslant 0$, then

$$
Z_{E, k}(s, x)=\sum_{j=0}^{|k|}\left\langle\begin{array}{c}
|k| \\
j
\end{array}\right\rangle \sum_{i=0}^{|k|-j}(-1)^{i}\left(\begin{array}{c}
|k|-j \\
i
\end{array}\right) \zeta_{E}(s-1, x-|k|+i) .
$$

Proof. (i) One computes

$$
\begin{aligned}
Z_{E, k}(s, x) & =\frac{2}{\Gamma(s)} \sum_{m=0}^{\infty} \frac{1}{(m+1)^{k}} \int_{0}^{\infty} \frac{\left(1-e^{-t}\right)^{m+1}}{1+e^{t}} e^{-x t} t^{s-2} d t \\
& =\frac{1}{s-1} \frac{2}{\Gamma(s-1)} \sum_{m=0}^{\infty} \frac{1}{(m+1)^{k}} \frac{e^{-(x+j) t}}{1+e^{t}} t^{s-2} d t
\end{aligned}
$$

which is the right-hand side by (4.1).

(ii) Since

$$
\operatorname{Li}_{k}\left(1-e^{-t}\right)=e^{(|k|+1) t} \sum_{j=0}^{|k|}\left\langle\begin{array}{c}
|k| \\
j
\end{array}\right\rangle\left(1-e^{-t}\right)^{|k|-j}
$$

by $(2.2)$, we have

$$
Z_{E, k}(s, x)=\sum_{j=0}^{|k|}\left\langle\begin{array}{c}
|k| \\
j
\end{array}\right\rangle \sum_{i=0}^{|k|-j}(-1)^{i}\left(\begin{array}{c}
|k|-j \\
i
\end{array}\right) \int_{0}^{\infty} \frac{e^{-(x+i-|k|-1) t}}{1+e^{t}} t^{s-2} d t
$$

This yields the formula.

\subsection{A relation with the Arakawa-Kaneko zeta function $Z_{B, k}(s, x)$}

We review the zeta function investigated in [3]. For $k \in \mathbb{Z}$ we set

$$
Z_{B, k}(s, x):=\frac{1}{\Gamma(s)} \int_{0}^{\infty} \frac{\operatorname{Li}_{k}\left(1-e^{-t}\right)}{1-e^{-t}} e^{-x t} t^{s-1} d t
$$

the Laplace-Mellin integral. It is defined for $\operatorname{Re}(s)>0$ and $x>0$ if $k \geqslant 1$, and for $\operatorname{Re}(s)>0$ and $x>|k|+1$ if $k \leqslant 0$. The function $s \mapsto Z_{B, k}(s, x)$ has analytic continuation to an entire function on the whole complex $s$-plane and

$$
Z_{B, k}(-n, x)=(-1)^{n} B_{n}^{(k)}(-x) .
$$

holds for $n \geqslant 0, x>0$.

There is a relation between $Z_{E, k}(s, x)$ and $Z_{B, k}(s, x)$.

Theorem 4.5. For $k \in \mathbb{Z}$, we have

$$
s Z_{E, k}(s+1, x)+s Z_{E, k}(s+1, x-1)=2 Z_{B, k}(s, x)-2 Z_{B, k}(s, x+1) .
$$


Proof. The left-hand side of the identity in the theorem equals

$$
\begin{aligned}
\frac{2}{\Gamma(s)} \int_{0}^{\infty} \frac{\operatorname{Li}_{k}\left(1-e^{-t}\right)}{t\left(1+e^{t}\right)} t\left(1+e^{t}\right) e^{-x t} t^{s-1} d t & \\
& =\frac{2}{\Gamma(s)} \int_{0}^{\infty} \frac{\operatorname{Li}_{k}\left(1-e^{-t}\right)}{1-e^{-t}}\left(1-e^{-t}\right) e^{-x t} t^{s-1} d
\end{aligned}
$$

which is nothing but the right-hand of the identity.

\section{Generalized poly-Euler polynomials and Arakawa-Kaneko type $L$-functions}

In this section, using a Dirichlet character, we extend poly-Euler polynomials and Arakawa-Kaneko type zeta functions.

\subsection{Generalized poly-Euler polynomials}

Let $f$ be a positive integer and $\chi$ the Dirichlet character with conductor $f=f_{\chi}$. As is well-known, generalized Euler polynomials are defined by the generating function

$$
2 \sum_{a=0}^{f-1}(-1)^{a} \chi(a) \frac{e^{a t}}{e^{f t}+1} e^{x t}=\sum_{n=0}^{\infty} E_{n, \chi}(x) \frac{t^{n}}{n !} .
$$

Definition 5.1. Let $k \in \mathbb{Z}$. We define generalized poly-Euler polynomials $E_{n, \chi}^{(k)}(x)$ $(n=0,1,2, \ldots)$ by

$$
\frac{2}{f} \sum_{a=0}^{f-1}(-1)^{a} \chi(a) \frac{\operatorname{Li}_{k}\left(1-e^{-f t}\right)}{t\left(e^{f t}+1\right)} e^{(x+a) t}=\sum_{n=0}^{\infty} E_{n, \chi}^{(k)}(x) \frac{t^{n}}{n !} .
$$

We call $E_{n, \chi}^{(k)}:=E_{n, \chi}^{(k)}(0)(n=0,1,2, \ldots)$ generalized poly-Euler numbers.

One can easily prove the following two theorems as in poly-Euler polynomial case.

Theorem 5.2 (Addition formula). For $k \in \mathbb{Z}, n \geqslant 0$, we have

$$
E_{n, \chi}^{(k)}(x+y)=\sum_{m=0}^{n}\left(\begin{array}{c}
n \\
m
\end{array}\right) E_{m, \chi}^{(k)}(x) y^{n-m} .
$$

Theorem 5.3 (Appell sequence). For $k \in \mathbb{Z}, n \geqslant 0$, we have

$$
\frac{d}{d x} E_{n+1, \chi}^{(k)}(x)=(n+1) E_{n, \chi}^{(k)}(x) .
$$

We have the following expressions of $E_{n, \chi}^{(k)}(x)$ in terms of $E_{n}^{(k)}(x)$ and $E_{n}(x)$. 
Theorem 5.4. For any $n \geqslant 0$, we have

$$
\begin{aligned}
E_{n, \chi}^{(k)}(x) & =f^{n} \sum_{a=0}^{f-1}(-1)^{a} \chi(a) E_{n}^{(k)}\left(\frac{x+a}{f}\right), \\
E_{n, \chi}^{(k)} & =f^{n} \sum_{a=0}^{f-1}(-1)^{a} \chi(a) E_{n}^{(k)}\left(\frac{a}{f}\right) .
\end{aligned}
$$

Theorem 5.5. For $k \in \mathbb{Z}, n \geqslant 0$, we have

$$
\begin{aligned}
& E_{n, \chi}^{(k)}(x) \\
& =\frac{f^{n}}{n+1} \sum_{a=0}^{f-1}(-1)^{a} \chi(a) \sum_{m=0}^{\infty} \frac{1}{(m+1)^{k}} \sum_{j=0}^{m+1}(-1)^{j}\left(\begin{array}{c}
m+1 \\
j
\end{array}\right) E_{n+1}\left(\frac{x+a-f j}{f}\right) .
\end{aligned}
$$

\subsection{Arakawa-Kaneko type $L$-functions}

Definition 5.6. For $k \in \mathbb{Z}$, define the $L$-series attached to $\chi$ by the Laplace-Mellin integral

$$
L_{E, k}(s, x, \chi)=\frac{2}{f} \sum_{a=0}^{f-1}(-1)^{a} \chi(a) \frac{1}{\Gamma(s)} \int_{0}^{\infty} \frac{\operatorname{Li}_{k}\left(1-e^{-f t}\right)}{t\left(e^{f t}+1\right)} e^{-(x-a) t} t^{s-1} d t .
$$

By Proposition 4.2, $L_{E, k}(s, x, \chi)$ is defined for $\operatorname{Re}(s)>1$ and $x>0$ if $k \geqslant 1$, and $\operatorname{Re}(s)>1$ and $x>|k|+1$ if $k \leqslant 0$. We call $L_{E, k}(s, x, \chi)$ the ArakawaKaneko type L-function. These functions include Arakawa-Kaneko and Hurwitz $L$-functions:

Theorem 5.7. One has

$$
L_{E, k}(s, x, \chi)=f^{-s} \sum_{a=0}^{f-1}(-1)^{a} \chi(a) Z_{E, k}\left(s, \frac{x-a}{f}\right) .
$$

Especially,

$$
L_{E, 1}(s, x, \chi)=f^{-s} \sum_{a=0}^{f-1}(-1)^{a} \chi(a) \zeta_{E}\left(s, \frac{x-a}{f}+1\right) .
$$

Furthermore, if $\chi=\chi_{0}$, the trivial character, then

$$
L_{E, 1}\left(s, x, \chi_{0}\right)=Z_{E, 1}(s, x)=\zeta_{E}(s, x+1) .
$$

Theorem 5.8. The function $s \mapsto L_{E, k}(s, x, \chi)$ has analytic continuation to an entire function on the whole complex s-plane and has the identity

$$
L_{E, k}(-n, x, \chi)=(-1)^{n} E_{n, \chi}^{(k)}(-x)
$$

for $n \geqslant 0, x>0$. 


\section{Proofs of theorems in Section 5}

Proof of Theorem 5.4. To begin with, let us prove the first identity. We make use of the generating function for $E_{n, \chi}^{(k)}(x)$. We calculate

$$
\begin{aligned}
\sum_{n=0}^{\infty}\left\{f^{n} \sum_{a=0}^{f-1}(-1)^{a} \chi(a) E_{n}^{(k)}\left(\frac{x+a}{f}\right)\right\} \frac{t^{n}}{n !} & =\sum_{a=0}^{f-1}(-1)^{a} \chi(a) \sum_{n=0}^{\infty} E_{n}^{(k)}\left(\frac{x+a}{f}\right) \frac{(f t)^{n}}{n !} \\
& =\sum_{a=0}^{f-1}(-1)^{a} \chi(a) \frac{2 \operatorname{Li}_{k}\left(1-e^{-f t}\right)}{f t\left(e^{f t}+1\right)} e^{\left(\frac{x+a}{f}\right) f t}
\end{aligned}
$$

which yields the generating function for $E_{n, \chi}^{(k)}(x)$. The second identity comes from the first identity for $x=0$.

Proof of Theorem 5.5. The result follows from Theorems 2.4 and 5.4.

Proof of Theorem 5.7. The first identity is proved by

$$
L_{E, k}(s, x, \chi)=\sum_{a=0}^{f-1}(-1)^{a} \chi(a) \frac{2}{\Gamma(s)} \int_{0}^{\infty} \frac{\operatorname{Li}_{k}\left(1-e^{-f t}\right)}{(f t)\left(e^{f t}+1\right)} e^{-\left(\frac{x-a}{f}\right) f t} t^{s-1} d t
$$

(put $u=f t)$

$$
=f^{-s} \sum_{a=0}^{f-1}(-1)^{a} \chi(a) Z_{E, k}\left(s, \frac{x-a}{f}\right) .
$$

Recalling (4.1), we obtain the second result.

Proof of Theorem 5.8. The first claim follows from Theorem 4.3, 5.7. As for the second claim, by Theorem 4.3, Definition 5.1 and Theorem 5.4,

$$
\begin{aligned}
L_{E, k}(-n, x, \chi) & =(-1)^{n} f^{n} \sum_{a=0}^{f-1}(-1)^{a} \chi(a) E_{n}^{(k)}\left(\frac{-x+a}{f}\right) \\
& =(-1)^{n} E_{n, \chi}^{(k)}(-x) .
\end{aligned}
$$

\section{Concluding remark}

Dilcher [8] and Chen [6] investigated the following sums of products of Euler polynomials:

$$
\sum_{\substack{i_{1}+\cdots+i_{m}=n \\
i_{1}, \ldots, i_{m} \geqslant 0}}\left(\begin{array}{c}
n \\
i_{1}, \ldots, i_{m}
\end{array}\right) E_{i_{1}}\left(x_{1}\right) \cdots E_{i_{m}}\left(x_{m}\right)
$$


for $n \geqslant 0$. It seems interesting to consider the following sums of poly-Euler polynomials:

$$
\sum_{\substack{i_{1}+\cdots+i_{m}=n \\
i_{1}, \ldots, i_{m} \geqslant 0}}\left(\begin{array}{c}
n \\
i_{1}, \ldots, i_{m}
\end{array}\right) E_{i_{1}}^{(k)}\left(x_{1}\right) \cdots E_{i_{m}}^{(k)}\left(x_{m}\right)
$$

for $n \geqslant 0$ and $k \in \mathbb{Z}$.

Acknowledgement. The author expresses his sincere gratitude to the referee for careful reading of the manuscript and many valuable suggestions. The author would like to thank A. Bayad for helpful discussion. He was supported by the Grant-in-Aid for Scientific Research (No. 20540026), from the Japan Society for the Promotion of Science.

\section{References}

[1] T. Arakawa and M. Kaneko, Multiple zeta values, poly-Bernoulli numbers, and related zeta functions, Nagoya Math. J. 153 (1999), 1-21.

[2] T. Arakawa and M. Kaneko, On poly-Bernoulli numbers, Comment. Math. Univ. St. Pauli 48 (1999), 159-167.

[3] A. Bayad and Y. Hamahata, Polylogarithms and poly-Bernoulli polynomials, Kyushu J. Math. 65 (2011), 15-24.

[4] A. Bayad and Y. Hamahata, Arakawa-Kaneko L-functions and generalized poly-Bernoulli polynomials, J. Number Theory 131 (2011), 1020-1036.

[5] A. Bayad and Y. Hamahata, Multiple polylogarithms and multi-poly-Bernoulli polynomials, Funct. Approx. Comment. Math. 46 (2012), 45-61.

[6] K.-W. Chen, Sums of products of generalized Bernoulli polynomials, Pacific J. Math. 208 (2003), 39-52.

[7] M.-A. Coppo and B. Candelpergher, The Arakawa-Kaneko zeta function, Ramanujan J. 22 (2010), 153-162.

[8] K. Dilcher, Sums of products of Bernoulli numbers, J. Number Theory 60 (1996), 23-41.

[9] R. L. Graham, D. L. Knuth, and O. Patashnik, Concrete Mathematics, 2nd ed., Addison-Wesley, Reading, MA,1994.

[10] Y. Hamahata and H. Masubuchi, Recurrence formulae for multi-polyBernoulli numbers, Integers 7 (2007), A46.

[11] Y. Hamahata and H. Masubuchi, Special multi-poly-Bernoulli numbers, J. Integer Sequences 10 (2007), article 07.4.1.

[12] M. Kaneko, Poly-Bernoulli numbers, J. Théorie de Nombres Bordeaux 9 (1997), 221-228.

[13] A. Laurinčikas and R. Garunkštis, The Lerch Zeta-function, Kluwer Academic Publishers, 2010.

[14] S. Roman, The Umbral Calculus, Academic Press, 1984.

[15] J.-W. Son and M.-S. Kim, On poly-Eulerian numbers, Bull. Korean Math. Soc. 36 (1999), 47-61.

[16] D. Zagier, Zetafunktionen und quadratische Körper, Springer. 
Address: Yoshinori Hamahata: Department of Applied Mathematics, Okayama University of Science, Ridai-cho 1-1, Okayama, 700-0005, Japan.

E-mail: hamahata@xmath.ous.ac.jp

Received: 5 September 2012; revised: 14 January 2014 